\title{
Low-Level Laser Therapy: "Western School" vs "Eastern School"
}

\author{
Sergey V. Moskvin ${ }^{1}$ \\ ${ }^{1}$ Academy of Postgraduate Education Under FSBU FSCC of FMBA of Russia, Russia
}

*Correspondence to: S.V. Moskvin, Academy of Postgraduate Education Under FSBU FSCC of FMBA of Russia, Russia, Email: 7652612@mail.ru

Received: April 8, 2021, Accepted: July 21, 2021, Published online: October 20, 2021

\section{Introduction}

Low-level laser therapy (LLLT) is one of the most common methods of physiotherapy, which is the modern stage in the development of heliotherapy and light therapy. At the end of the 19th century, Nobel laureate N.R. Finsen proved that it is possible to significantly increase the effectiveness of light therapy by using "special" lamps instead of sunlight so that one is able to control their power, area and time of exposure with the ability to allocate the desired spectrum of light. In the early 1960s, lasers appeared on the market. They were sources of monochromatic light (and therefore, it was not necessary to use light filters to isolate a part of the spectrum), whose energy parameters were much easier to control. These qualities led to the emergence of a fundamentally new direction - LLLT, which is characterized by its higher efficiency and universality.

Exposure to low-intensity laser illumination (LILI) causes a reaction in the body, and as a result, homeostasis (which was disturbed beforehand) is restored, resulting in the recovery of the patient. After absorption of laser light in the cells, $\mathrm{Ca}^{2+}$-dependent processes are the first to activate, launching numerous secondary reactions at the tissue and organism level (Figure 1). ${ }^{1}$

LLLT is used successfully in almost all areas of modern medicine: obstetrics and gynecology; andrology and urology; neurology; otorhinolaryngology; pediatrics; dentistry; musculoskeletal diseases, joint and muscle pain; etc.

Correlated and combined methods of laser therapy are developing quite rapidly, such as laser phoresis, ${ }^{2}$ laservacuum massage and High Frequency laser therapy.

The development of medicine in the context of globalization is impossible without the exchange of information coming from different countries. This allows specialists not only to get acquainted with new medical technologies but also to more objectively evaluate their own achievements. If some methods allow getting better results, then you need to try them, especially if the limitations of the "conventional" treatment regimens are known. Conservatism is necessary in medicine as it is anywhere, but the artificial limitation by the framework of the customary does not allow its development.

Many treatment methods offered by specialists from different countries are quite unique as they have been developed in conditions of political and linguistic isolation. From the point of view of their authors, the good results which are demonstrated by them, and even the remarkable results of the treatment, allow the technique to be distributed automatically throughout the world. However, this opinion is only based on many years of successful experience of practical application, even in several clinics, but not on the data of standardized studies. In addition, in most cases, there is no theoretical justification, an explanation of the mechanisms of therapeutic action. Therefore, their value for the science world and medical practice is often questioned.

On the other hand, the rejection of unusual (nonstandard, non-traditional) methods of treatment, in turn, is almost always justified by phrases such as "I do not believe" and "this cannot be", rather than scientific (objective) evidence of an absence or presence of a therapeutic effect. Once upon a time LLLT was referred to as "voodoo medicine"3 in the 1980s-1990s, conferences did not accept reports on the therapeutic use of lasers, and we listened to the ridicule from foreign colleagues. While laser surgery was easily accepted because everything was "clearly understood", laser therapy was denied even with the theoretical possibility that LILI may have any influence on a living organism. ${ }^{4}$

In addition to subjective factors, there are two main objective reasons for this mistrust. The first of them is, in many aspects, the fundamental differences in scientific medical education. 


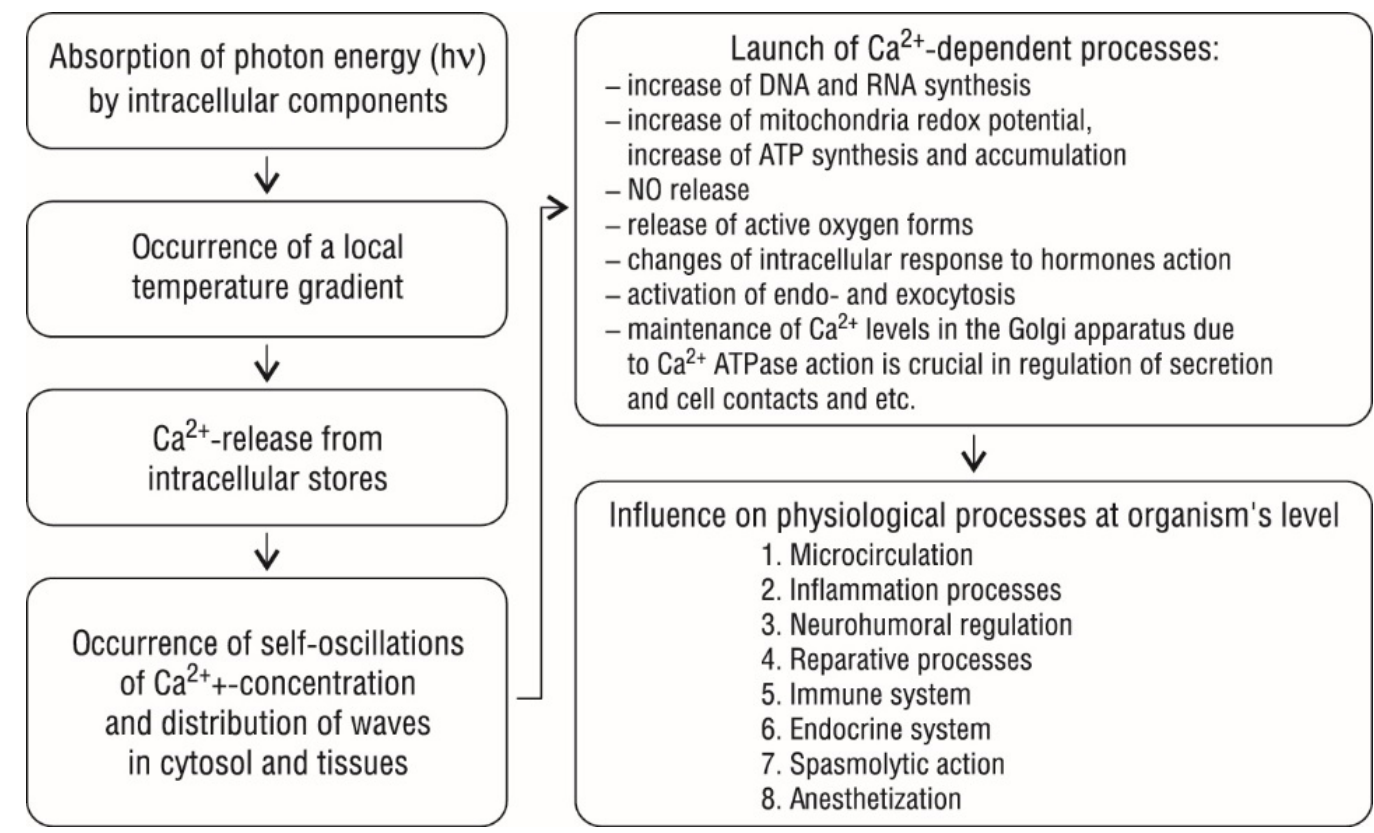

Figure 1. The Sequence of the Developing Biological Effects of Laser Exposure

One approach can be generally referred to as the "Western School" (WS), where students learn more about the specifics of everything and "compartmentalize" a person into organs, specialized cells, cell organoids, molecules and atoms. Accordingly, diseases are classified by organs and targets that have been pathologically affected, and almost all treatments are based on the principle of substitution and external compensation. For example, when a tooth has fallen out, the necessary way to treat this is to implant an artificial tooth. The body cannot cope with an infection; antibiotics must be used to kill the bacteria.

This approach has been developed over several centuries in Europe, although not without professionals opposing this in favour of another point of view. Even at the stage of the formation and development of modern medical science in WSs, there were fierce debates about the approaches to the study of structures and principles of the existence of living objects, as well as the fundamental basis of treatment, which was perfectly reflected in the immortal work of the great German poet ${ }^{5}$ :

He who would study organic existence, First drives out the soul with rigid persistence; Then the parts in his hand he may hold and class, But the spiritual link is lost, alas!

If you study only the "structure" of a living being, what it consists of, without constantly correlating the gained knowledge with the general laws of the functioning of the biological system as a whole (that is, without studying life as such), then there is very little useful information which is learned. This situation was described very accurately by the remarkable American science fiction writer Harry Harrison: "Facts for them were always hooked up in a series. Whereas in truth they had to be analyzed as a complex circuit with elements like positive and negative feedback, and crossover switching. It's little wonder they did do badly."

Nevertheless, it is necessary to admit the obvious that WS has given the world thousands and thousands of highly effective treatment technologies. The countries that use these technologies have not only the longest life expectancy, but more importantly, the quality of life is high, the older generation lives fully, and does not only live out their day. This is all thanks to science and research, conducted by scientists of various profiles.

The second direction is the "Eastern School" (ES), in which a patient is treated as a whole and is not treated based solely on the disease, more often using nonspecific methods of treatment, and at the core of their restoration is the disturbed ability of the body to protect and normally regulate various physiological processes. For example, instead of antibiotics, we restore the body's ability to normalize (activate) the patients' own human immune system.

If in the WS more studies examine the "device" of living, what the bio-object consists of and how the different parts interact with each other, the ES studies the "function" of the living system as a whole and the laws of its response to external influence. For ES, the word "science" has a special meaning, the response of the biological system to external influences is studied, primarily from the position of sanogenesis, i.e., the ability of the living organism to independently fight the disease by activating internal functional reserves.

The most striking example of medical technology in this area is acupuncture, in which, for therapeutic 
purposes, effects are exerted on certain points on the human or animal body, causing the necessary response of the organism. These "biologically active" points and the necessary sequence of effects on them were determined empirically. These local zones are not morphologically different from surrounding tissues and the mechanism of healing is unknown, there are not even any theoretical ideas. Despite this fact, the method is quite common due to the effectiveness in most cases.

One of the problems for ES is that its representatives very often use non-standardized terms that only they themselves understand to describe their medical technologies, and they do not bother explaining the mechanisms or scientific justification of how all this "works". For example, acupuncture uses concepts such as the Five Element System (Fire, Earth, Metal, Water and Wood) and Meridians. It is quite understandable that such an approach often causes rejection by WS specialists.

Recently, due to the clearly observed tendency to blur the borders between East and West, the ES's views are gaining an increasing understanding among Western doctors. For example, in English, there are quite a few scientific journals on acupuncture that have gained the attention of many readers and a high enough IF (impact factor) (for example, Acupuncture in Medicine, http://aim. bmj.com). Currently, laser acupuncture is more actively used. $^{7}$

In our opinion, in each of the disciplines, both WS and ES disciplines have their pros and cons, but one must take all the good and ignore all the bad in both schools.

LLLT is a vivid example of highly effective medical technology. Unlike many other therapeutic methods of the East (including acupuncture), we have rigorously demonstrated the mechanisms (primary and secondary see Figure 1) underlying the method, the understanding of which, in turn, allows us to not only explain the numerous experimental and clinical data but also substantiate the most effective parameters of treatment methods, as well as ways to optimize them. In LLLT, when describing techniques, only standard terms and definitions are used that are familiar to all Western representatives. The effectiveness of laser therapy has been proven in the course of thousands of clinical studies.

LLLT is a perfect example of a close interaction between WS and ES specialists, which has resulted in the birth of a new, remarkable, extremely effective, simple and reproducible treatment that has no side effects and contraindications in many diseases; a large part of the diseases according to the WS canons with the use of standard WS schemes are "not treatable".

Of course, it is necessary to apply LLLT correctly, and it is very easy to do this. We have developed strict recommendations, standard methods and principles for their optimization, which allows specialists of all types to treat their patients easily and effectively. ${ }^{8}$
1. For LLLT only lasers can be used, not light bulbs or LEDs.

2. In the methodology it is necessary to consistently set all parameters: wavelength, operating mode (continuous, modulated, pulsed), laser light power (average or pulsed), frequency for pulsed or modulated modes, exposure, localization, number of procedures per course. The area of illumination (and the energy density) is distinguished by special nozzles. If even one of the parameters is set incorrectly, the technique will not be implemented and the effect will not be reached.

3. All the parameters should be selected with optimal precision; for example, increasing the laser power does not always lead to an improvement in the result, and it often becomes worse.

4. When the frequency is changed for pulsed lasers, the average power also varies proportionally. This allows you to select the optimal energy parameters, which are not active in continuous and modulated modes.

5. It is strictly forbidden to illuminate one zone (point) for more than five minutes, and the total time of the procedure should not exceed 20 minutes!

The $2^{\text {nd }}$ principle requires some clarification. In laser therapy, there is no such parameter as "dose" or "dosage". There is "energy" = power $\times$ time [J] and "energy density" = energy/area $\left[\mathrm{J} / \mathrm{cm}^{2}\right]$. The mathematical demonstrations are absolutely not necessary. For instance, the ED may be the same (most often the optimal $1 \mathrm{~J} / \mathrm{cm}^{2}$ ) in three different situations (assuming a contact-mirror technique and an effective area of $1 \mathrm{~cm}^{2}$ ):

1. the power of $1 \mathrm{~mW}$ is multiplied by the exposure time of 1000 seconds (about 15 minutes) $=1 \mathrm{~J} / \mathrm{cm}^{2}$;

2. the power of $1000 \mathrm{~mW}$ is multiplied by the exposure time of 1 second $=1 \mathrm{~J} / \mathrm{cm}^{2}$;

3. the power of $10 \mathrm{~mW}$ is multiplied by the exposure time of 100 seconds (about 1.5 minutes) $=1 \mathrm{~J} / \mathrm{cm}^{2}$.

However, the effect, i.e., a positive result of the treatment, will be ONLY in the 3rd case, when all the optimal parameters are set, and even then, only for lasers of continuous operation with a wavelength of $635 \mathrm{~nm}$ (red spectrum). In options 1 and 2, there will be no curative effect for any laser or mode of operation.

There are other recommendations that specialists in Russia are studying at specialized courses.

Analysis of different periods of development of LLLT allows us to conclude that the capabilities of LLLT are still inadequately used, and there are all prerequisites for the active development and expansion of the application. This opinion is supported by the fact that only in 2016 more articles were published in English-language journals than in the whole period from 1965 (the first reports) until 2000. Despite the fact that in the overwhelming majority of cases, the quality of publications is extremely low (this fact deserves a separate and detailed discussion), 
the positive trend is encouraging.

\section{Conclusion}

Thus, LLLT, on the one hand, is the method of Eastern medicine since the effect is carried out on the whole person and the initial response reactions of the body are considered as the main mechanism of the biomodulating action of LILI. On the other hand, LLLT complies with all the rules of Western medicine since the mechanisms of the biomodulating action of LILI are well understood and are considered exclusively in terms of the classical sciences - anatomy, physiology, biochemistry, and so on.

Eastern and Western medicine should not be opposed but used synergistically; each direction has its own advantages, while LLLT, combining these methodologies, achieves all the possibilities of the full potential of treatment.

\section{Ethical Considerations \\ Not applicable.}

\section{Conflict of Interests}

The authors declare that they have no conflict of interest.

\section{References}

1. Moskvin SV. Low-Level Laser Therapy in Russia: History, Science and Practice. J Lasers Med Sci. 2017;8(2):56-65. doi: 10.15171/jlms.2017.11

2. Moskvin SV, Konchugova TV. [The rationale for the application of laserophoresis of biologically active compounds]. Vopr Kurortol Fizioter Lech Fiz Kult. 2012 Sep-Oct;(5):57-63. [Russian]

3. Coulter AH. Low-energy laser research in wound healing. J Clin Laser Med Surg.1994;12(2):117-118.

4. Berlien HP, Müller G. Applied Laser Medicine. SpringerVerlag Berlin Heidelberg; 1989. Doi:10.1007/978-3-64218979-1

5. Goethe JW. Faust: a tragedy. Translated, in the original metres, with copious notes, by Bayard Taylor. London Ward, Lock; 1890.

6. Harrison H. The K-Factor. Positronic Publishing; 2015.

7. Moskvin SV, Agasarov LG. Laser acupuncture: 35 years of successful application in Russia (narrative review). J Lasers Med Sci. 2020;11(4):381-389. doi:10.34172/jlms.2020.61.

8. Moskvin SV. Low-level laser therapy and light energy. Photobiomodul Photomed Laser Surg. 2019;37(5):267-268. doi:10.1089/photob.2019.4622. 